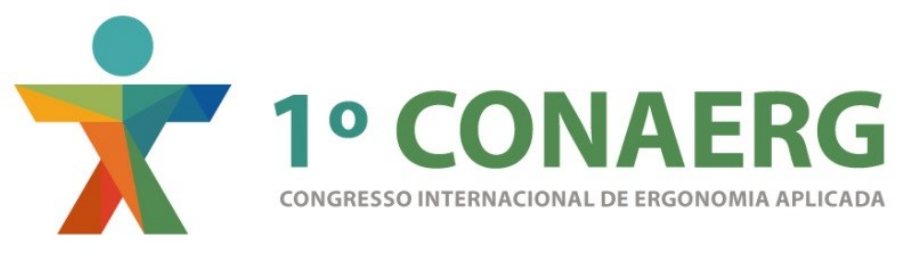

\title{
EFEITOS DE UMA INTERVENÇÃO ERGONÔMICA, BASEADA NO AJUSTE DOS POSTOS DE TRABALHO EM RELAÇÃO AO ESTRESSE, FADIGA, BEM ESTAR E SINTOMAS MUSCULOESQUELÉTICOS
}

\author{
Fernanda Cabegi de Barros (1)
}

Raiane Fabiola de Azevedo (2)

Tatiana de Oliveira Sato (3)

(1) Universidade Federal de São Carlos, Doutoranda no Programa de Pós-Graduação em Fisioterapia

e-mail: barros.fernandac@gmail.com

(2) Universidade Federal de São Carlos, Graduanda de Fisioterapia

e-mail: raiane azevedo06@hotmail.com

(3) Universidade Federal de São Carlos, docente do Departamento de Fisioterapia e do Programa de Pós-Graduação em Fisioterapia

e-mail: tatisato@ufscar.br

\begin{abstract}
RESUMO
O objetivo deste estudo foi avaliar os efeitos de uma intervenção ergonômica baseada no ajuste dos postos de trabalho em relação ao estresse, fadiga, bem estar e sintomas musculoesqueléticos. Trinta trabalhadores de escritório foram divididos em dois grupos (experimental [GE] e controle [GC]) e comparados antes e 6 meses após a intervenção, por meio de questionários. O GC não recebeu nenhuma intervenção. O GE apresentou melhora, estatisticamente não significativa, dos sintomas musculoesqueléticos, fadiga e demanda de trabalho. Embora os efeitos benéficos da intervenção tenham sido observados, ainda são necessários estudos sobre esta temática envolvendo maior número de trabalhadores.
\end{abstract}

\section{ABSTRACT}

The aim of this study was to evaluate the effects of an ergonomic intervention based on the furniture adjustment in relation to stress, fatigue, well-being and musculoskeletal symptoms. Thirty office workers were divided into two groups (experimental [EG] and control [CG]) and compared before and 6 months after the intervention through questionnaires. The CG received no intervention. GE has improved musculoskeletal symptoms, fatigue and work demands, but it was not statistically significant. Although the beneficial effects of the intervention have been observed, further studies are needed on this subject with larger number of workers. 


\section{INTRODUÇÃO}

O trabalho em escritório é, em geral, associado ao uso de computadores e à postura sentada mantida por tempo prolongado (BERNARD, 1997; CALLAGHAN, 2010; BRINK e LOUW, 2013; MÖRL e BRADL, 2013). O trabalho sentando era considerado eficaz no sentido de promover melhor rendimento e menor fadiga (GRANDJEAN, 1998). As justificativas para tais vantagens seriam explicadas, principalmente, pelo baixo consumo energético (COURY, 1994). No entanto, a postura sentada favorece a ocorrência de problemas na coluna, associados à fraqueza dos músculos abdominais e do tronco. Além disso, há associações de lesões musculoesqueléticas de pescoço com maior experiência de trabalho, alta sobrecarga muscular e exigências cognitivas sem pausas adequadas (KALINIENE et al 2013).

Somado aos distúrbios musculoesqueléticos, o trabalho de escritório também está associado à grande demanda psicológica (AMICK, 2003; MACDONALD et al 2001; KAUSTO et al 2011). De acordo com Westgaard e Winkel (2011), existe relação entre fatores psicossociais adversos e o desenvolvimento de síndromes dolorosas musculares. Sabe-se que a cintura escapular está mais susceptível a mialgias devido ao estresse mental, possivelmente devido a sensibilidade existente do músculo trapézio à tensão psicossocial (BART, 2008; WEESTGAARD e WINKEL, 2011).

Dessa forma, a prevalência de doenças musculoesqueléticas relacionadas ao trabalho indica a necessidade de propostas efetivas de prevenção e controle, e voltadas a ajuste dos postos de trabalho (MINISTÉRIO DA SAÚDE DO BRASIL, 2001). Neste contexto, a Norma Regulamentadora 17 (NR17) indica alguns parâmetros para a adaptação das condições de trabalho aos trabalhadores, de modo a proporcionar conforto, segurança e desempenho eficiente (MINISTÉRIO DO TRABALHO E EMPREGO, 2008).

Dentro desse contexto, é possível utilizar métodos de avaliação para quantificar os efeitos destas intervenções. Estes métodos incluem ferramentas como questionários e escalas, que incluem frequência, localização e intensidade dos sintomas, bem como a capacidade funcional dos trabalhadores (ALONSO et al 1990; DEYO et al 1998; POBLETE et al 2012).

Dentre os questionários disponíveis, o Questionário Nórdico de Sintomas Osteomusculares (QNSO) avalia os sintomas musculoesqueléticos e é considerado válido e útil como ferramenta de avaliação e mensuração de ganhos com possíveis intervenções (ANDERSON et al 1987; BJORKESTEN et al 1999; CRAWFORD, 2007; TAKEKAWA et al, 2015).

Vale ressaltar que as intervenções ergonômicas devem ser implementadas de acordo com as necessidades de cada trabalhador, levando em consideração os fatores biomecânicos e organizacionais (GONÇALVES, 1995; KALINIENE et al., 2013).

Uma vez que os aspectos psicossociais no trabalho estão associados aos sintomas (COSTA et al; 2010; RIJN et al; 2010; YUE et al., 2014), é plausível a ideia de que o ajuste no posto de trabalho possa melhorar os indicadores de estresse, fadiga, bem estar, além dos próprios sintomas musculoesqueléticos.

Assim, o objetivo deste estudo foi avaliar a eficácia de uma intervenção ergonômica baseada no ajuste dos postos de trabalho em relação ao estresse, fadiga, bem estar e sintomas musculoesqueléticos em trabalhadores de escritório.

\section{MATERIAL E MÉTODOS}

\subsection{Local do estudo}

Este estudo foi realizado em uma Secretaria responsável pela Educação à Distância de uma Instituição de Ensino Superior, na qual trabalham 60 funcionários. A Secretaria é dividida em setores de administração, gestão de pessoas, finanças, relações institucionais, coordenação 
pedagógica, desenvolvimento e aperfeiçoamento profissional, inovações em tecnologias na educação.

\subsection{Participantes}

Foram avaliados 30 trabalhadores com idade entre 18 e 60 anos, que tinham rotina de trabalho em escritório de pelo menos $4 \mathrm{~h} / \mathrm{dia}$ em 5 dias da semana, os quais concordaram em participar do estudo assinando o termo de consentimento livre e esclarecido. Os critérios de exclusão do estudo foram: IMC maior que $30 \mathrm{~kg} / \mathrm{m}^{2}$, não ter posto de trabalho fixo ou compartilhar o posto com outro trabalhador, usar notebook, usar dois monitores e ter realizado cirurgia nos últimos 6 meses. A alocação dos sujeitos aos grupos controle (GC) e experimental (GE) foi realizada de forma aleatorizada por salas (unidade de agrupamento ou clusters).

O projeto de pesquisa foi aprovado pelo Comitê de Ética e Pesquisa com Seres Humanos da Universidade (CAAE: 31938414.2.0000.5504, parecer N. 794.522, ANEXO I).

\subsection{Equipamentos e Instrumentos}

Foi aplicado um questionário online sobre dados pessoais, dominância manual, nível de escolaridade, histórico ocupacional, presença de dor ou desconforto físico e hábitos de vida. Ainda para caracterização da amostra foram aplicados os questionários:

- Questionário Internacional de Atividade Física (IPAQ): utilizado para determinar o nível de atividade física por meio do tempo gasto em atividades físicas semanalmente (PARDINI et al., 2001; BENEDETTI et al., 2007).

- Questionário de dependência de nicotina (Fagerström): aborda questões sobre o tempo e quantidade de cigarros, vontade de fumar, entre outras. O questionário apresenta níveis aceitáveis de consistência interna e foi traduzido e validado para o português (HEATHERTON et al., 1991).

- $\quad$ Teste de Identificação de desordens devido ao uso do álcool (AUDIT): utilizado para avaliar o padrão do uso do álcool, identificando indivíduos que necessitem de intervenção. $O$ AUDIT é considerado fidedigno e válido (DONOVAN et al., 2006).

Os seguintes questionários foram aplicados para fins de quantificar mudanças em relação à intervenção:

- Questionário Nórdico de Sintomas Osteomusculares - QNSO (BARROS E ALEXANDRE, 2003), avalia de forma segmentar as regiões do corpo que podem apresentar sintomas, persistência da dor e influência da mesma em atividades de vida diária (PUGH et al, 2015).

- Bem estar no Trabalho: este questionário avalia o bem estar do trabalhador em relação ao seu trabalho com relação a um estado mental positivo e realizador sendo composto por vigor, concentração e dedicação. A classificação é em muito alto, alto, médio, baixo e muito baixo (GOULART et al., 2012; REETA et al., 2011)

- Escala de Estresse no Trabalho: Avalia o estresse no trabalho resultante da interação entre as demandas psicológicas, controle no processo de trabalho e apoio social recebido de colaboradores e chefes (ALVES, 2004). Assim, pessoas expostas a trabalhos com alta demanda e baixo controle, considerados de alto desgaste, apresentam as reações mais adversas de desgaste psicológico, tais como fadiga, ansiedade, depressão e enfermidade física (ALVES, 2004). O trabalho pode ser considerado também como passivo e ativo, e ainda, de baixo desgaste (ALVES, 2004).

- $\quad$ Escala de Necessidade de Descanso (ENEDE): questionário sensível aos sintomas imediatos da fadiga, efeitos cumulativos do trabalho e está relacionado a maiores índices de acidentes de trabalho e problemas de saúde (DE CROON et al., 2003; SWAEN et al.,2003). 
O ponto de corte de 45 pontos (KISS et al., 2008), é usado para classificar a necessidade de descanso em alta e baixa.

\subsection{Protocolo de Avaliação}

A unidade de agrupamento foi a sala na qual o sujeito trabalhava, assim todos os trabalhadores de uma mesma sala foram alocados em um mesmo grupo. Este procedimento visou minimizar o efeito de "contaminação" entre os grupos, assim evitou-se que o trabalhador identificasse mudanças no posto de trabalho de um colega de sala e adaptasse seu posto sem orientações adequadas.

Um link contendo os questionários foi encaminhado via e-mail para cada trabalhador. Após o preenchimento a intervenção nos postos de trabalho dos trabalhadores do grupo experimental foi iniciada. Após 6 meses, o questionário foi reenviado, via e-mail, para cada trabalhador de ambos os grupos.

\subsection{Intervenção Ergonômica nos Postos de Trabalho}

Os postos de trabalho foram ajustados conforme as recomendações ergonômicas disponíveis (AHONEM et al., 1989; Manual NR-17, 2002; PANAGIOTOPOULOU et al., 2003). Para a adequação do posto as medidas do mobiliário foram relacionadas às medidas antropométricas. O ajuste da altura da cadeira foi realizado pela regulagem da altura quando a cadeira era ajustável ou foi realizada a substituição da cadeira por uma de altura ajustável. Como o ajuste da cadeira visa elevar a altura do cotovelo em relação ao chão, em alguns casos, foi necessário utilizar apoio de pés.

Os apoios foram confeccionados com medidas distintas para que se adequasse a cada indivíduo. Assim, após adequar a altura da cadeira e o apoio dos pés, a altura do monitor foi ajustada, de forma que o nível de visão estivesse sobre o terço superior da tela do monitor (COURY, 1994). A regulagem da altura do monitor foi feita pela utilização de suportes, confeccionados em diferentes alturas de forma a atender as necessidades de cada trabalhador. Quanto ao posicionamento do monitor, o mesmo foi posicionado diretamente a frente do trabalhador (SONNE et al., 2012). Os valores preconizados para a distância do monitor à visão são de 40 e $75 \mathrm{~cm}$ (SONNE et al., 2012). O teclado e mouse foram posicionados em uma distância que permitiu apoio do antebraço sobre a mesa de trabalho e o mouse foi alinhado com o ombro e posicionado próximo do teclado (SONNE et al., 2012).

Além disso, a adequação dos postos de trabalho foi acompanhada pelo trabalhador e a melhor forma de obter os ajustes necessários foi discutida previamente com ele. $O$ trabalhador recebeu orientações sobre a importância e necessidade dos ajustes realizados em seu posto.

\subsection{Análise dos dados}

\section{Variáveis dependentes e independentes}

As variáveis dependentes avaliadas foram estresse, fadiga, bem estar e sintomas musculoesqueléticos. As variáveis independentes serão os grupos de comparação (GE vs GC) e o tempo (pré vs pós intervenção).

\section{Testes estatísticos}

Os dados foram analisados de maneira descritiva por meio da comparação entre os grupos, utilizando medidas de tendência central, variabilidade e intervalos de confiança. O teste de Mann Whitney foi usado para comparar os grupos em relação aos desfechos de interesse. Os dados foram analisados no programa SPSS e o nível de significância adotado foi de $5 \%$. 


\section{RESULTADOS}

Os dados pessoais e demográficos dos trabalhadores de ambos os grupos estão apresentados na Tabela 1. Nota-se que as médias de idade, massa corporal, altura, IMC e carga horária diária de trabalho foram similares entre os grupos. Os grupos diferiram discretamente em relação ao tempo de trabalho na empresa. Ambos os grupos apresentam uma proporção discretamente maior de mulheres e em ambos os grupos mais da metade dos trabalhadores possuem pós-graduação completa ou incompleta. Quanto à presença de sintomas de dor, ambos os grupos apresentam alta proporção de trabalhadores sintomáticos, em torno de $80 \%$. Em relação à prática de exercício físico regular, os grupos foram similares, com aproximadamente $40 \%$ de trabalhadores ativos. O consumo diário de álcool e cigarro foi baixo em ambos os grupos (<15\%)

Tabela 1. Dados pessoais e demográficos dos trabalhadores do grupo controle (GC) e experimental (GE).

\begin{tabular}{|c|c|c|}
\hline & $\mathrm{GC}(\mathrm{n}=15)$ & GE $(n=15)$ \\
\hline Idade (anos) [média (DP)] & $29,4(8,4)$ & $28,3(4,0)$ \\
\hline Massa corporal (kg) [média (DP)] & $68,2(14,8)$ & $68,4(9,3)$ \\
\hline Altura $(\mathrm{cm})$ [média (DP)] & $166,7(10,8)$ & $169,3(10,4)$ \\
\hline Índice de massa corporal $\left(\mathrm{kg} / \mathrm{m}^{2}\right)$ [média (DP)] & $24,3(3,6)$ & $23,8(1,4)$ \\
\hline Carga horária diária de trabalho [média (DP)] & $7,8(0,5)$ & $7,6(1,6)$ \\
\hline Tempo de trabalho na empresa (meses) [média (DP)] & $37,3(25,1)$ & $32,7(19,0)$ \\
\hline \multicolumn{3}{|l|}{ Sexo $[n(\%)]$} \\
\hline feminino & $9(60,0)$ & $8(53,4)$ \\
\hline masculino & $6(40,0)$ & $7(46,6)$ \\
\hline \multicolumn{3}{|l|}{ Escolaridade [n (\%)] } \\
\hline cursando graduação & $3(20,0)$ & $1(6,7)$ \\
\hline graduação completa & $4(26,7)$ & $4(26,7)$ \\
\hline cursando pós graduação & $5(33,3)$ & $6(40,0)$ \\
\hline pós graduação completa & $3(20,0)$ & $4(26,7)$ \\
\hline Presença de sintomas de dor [n (\%)] & $13(86,7)$ & $12(80,0)$ \\
\hline Realiza atividade física [n (\%)] & $7(46,6)$ & $6(40,0)$ \\
\hline Tabagismo [n (\%)] & $2(13,3)$ & $1(6,7)$ \\
\hline Consumo de álcool [n (\%)] & $2(13,3)$ & $2(13,3)$ \\
\hline
\end{tabular}

Na Tabela 2 pode-se observar os resultados dos questionários de estresse, fadiga e bem estar no trabalho para ambos os grupos antes e depois da intervenção.

Tabela 2. Resultados da escala de estresse, fadiga e bem estar no trabalho para os grupos controle (GC) e experimental (GE) antes (pré) e depois (pós) da intervenção. Os dados estão apresentados em média (DP).

GC $(n=13)$




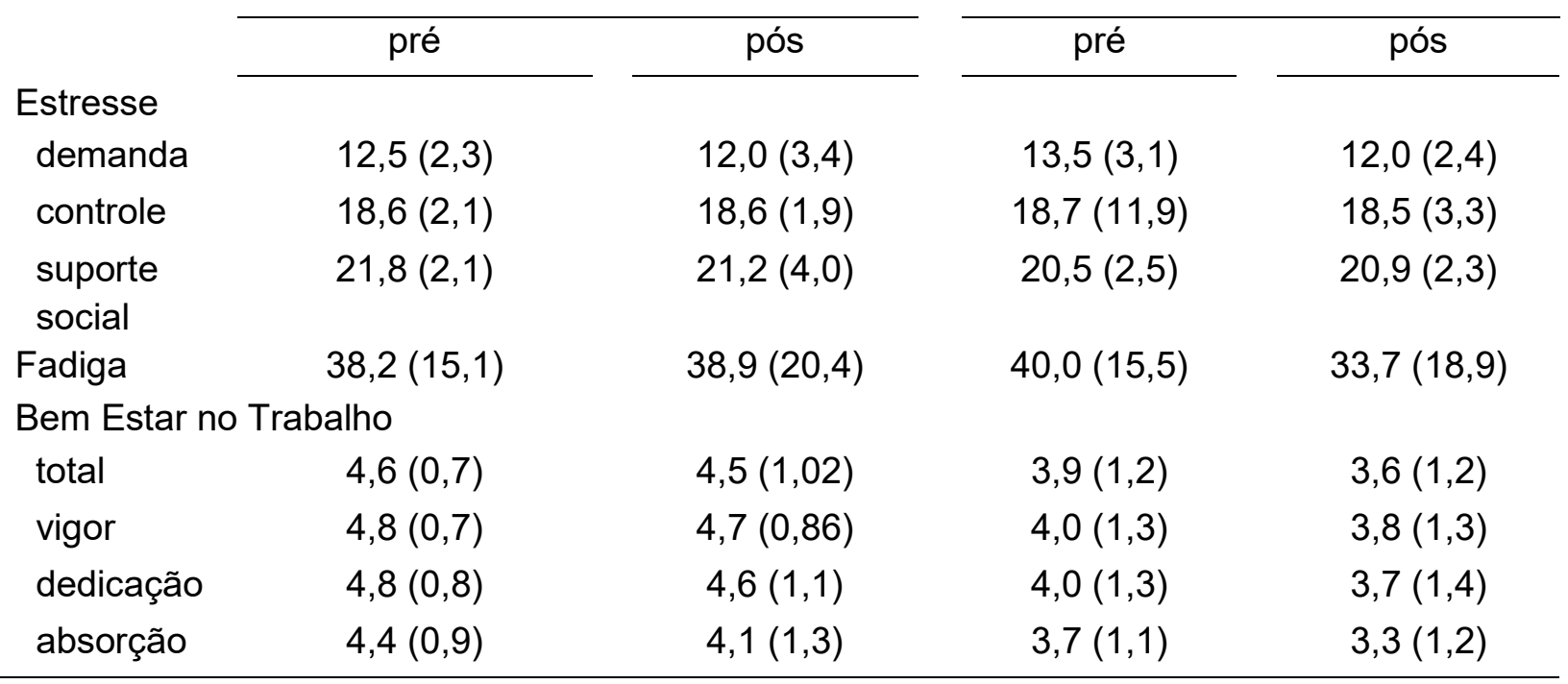

Em relação ao estresse, não houve diferença para os domínios controle $(P=0,79)$ e suporte social $(P=0,82)$ entre os grupos, já em relação à demanda observou-se uma redução, estatisticamente não significativa $(P=0,26)$, após a intervenção no $G E$. Houve também uma redução, não significativa $(P=0,27)$, da fadiga após a intervenção no $G E$. Em relação ao bem estar observou-se que tanto para a pontuação total $(P=1,00)$, como para os domínios vigor $(P=0,90)$, dedicação $(P=0,90)$ e absorção $(P=0,75)$ as médias reduziram do pré para o pós em ambos os grupos e esta variação não foi estatisticamente significativa.

A Tabela 3 mostra os resultados do Questionário Nórdico para as regiões de pescoço, ombros, coluna torácica e lombar, considerando os últimos sete dias.

Tabela 3. Questionário Nórdico de Sintomas Osteomusculares por região nos últimos sete dias. Os dados estão apresentados em $n(\%)$.

Geral

pioraram

melhoraram

iguais

Pescoço

pioraram

melhoraram

iguais

Ombros

pioraram

melhoraram

iguais

Coluna torácica

pioraram

melhoraram

iguais

Coluna lombar

\begin{tabular}{|c|c|c|c|}
\hline \multicolumn{2}{|c|}{$\mathrm{GC}(n=15)$} & \multicolumn{2}{|c|}{ GE $(n=15)$} \\
\hline pré & pós & pré & pós \\
\hline \multirow[t]{4}{*}{$13(86,6)$} & $13(86,6)$ & $12(80,0)$ & $7(46,6)$ \\
\hline & $2(13,3)$ & & $0(0,0)$ \\
\hline & $2(13,3)$ & & $5(33,4)$ \\
\hline & $11(73,3)$ & & $10(66,6)$ \\
\hline \multirow[t]{4}{*}{$4(26,6)$} & $7(46,6)$ & $3(20,00)$ & $2(13,33)$ \\
\hline & $6(40,0)$ & & $0(0,0)$ \\
\hline & $3(20,0)$ & & $1(6,6)$ \\
\hline & $6(40,0)$ & & $14(93,4)$ \\
\hline \multirow[t]{4}{*}{$5(33,3)$} & $5(33,3)$ & $2(13,3)$ & $2(13,3)$ \\
\hline & $3(20,0)$ & & $0(0,0)$ \\
\hline & $3(20,0)$ & & $0(0,0)$ \\
\hline & $9(60,0)$ & & $15(100,0)$ \\
\hline \multirow[t]{4}{*}{$6(40,0)$} & $6(40,0)$ & $5(33,3)$ & $1(6,6)$ \\
\hline & $6(40,0)$ & & $0(0,0)$ \\
\hline & $4(26,6)$ & & $4(26,6)$ \\
\hline & $5(33,3)$ & & $11(73,3)$ \\
\hline $7(46,6)$ & $5(33,3)$ & $4(26,6)$ & $3(20,0)$ \\
\hline
\end{tabular}


pioraram

$0(0,0)$

melhoraram

Os resultados do QNSO indicaram que no GC houve uma proporção de 6,6 a 40\% de trabalhadores que pioraram no período pré e pós intervenção; já no GE não houve piora nos sintomas musculoesqueléticos para todas as áreas avaliadas. Considerando uma visão geral, antes da intervenção 13 sujeitos do GC apresentaram dor em alguma região do corpo nos últimos sete dias, e passados seis meses, 13 trabalhadores do GC ainda apresentavam algum sintoma. No GE, 12 trabalhadores apresentavam sintomas antes e 7 apresentavam algum sintoma seis meses depois, ou seja, $33,4 \%$ dos trabalhadores do GE melhoraram em comparação a $13,3 \%$ do GC.

\section{DISCUSSÃO}

A intervenção realizada nos postos de trabalho foi eficaz para reduzir a percepção da dor no pescoço, ombros e região superior das costas, e também foi capaz de reduzir os níveis de fadiga captado pela Escala de Necessidade de Descanso (ENEDE). Entretanto para os níveis de estresse e bem-estar no trabalho, não foram alterados pela intervenção realizada no mobiliário.

Esperava-se que a intervenção apresentasse resultados positivos, melhorando na percepção do estresse, fadiga, bem-estar e sintomas musculoesqueléticos no grupo intervenção em relação ao grupo controle. Assim, os achados confirmaram parcialmente nossa hipótese inicial, uma vez que em relação a escala de estresse, apenas o domínio da demanda apresentou melhora, e em relação ao bem-estar, ao contrário do esperado, todos os seus domínios apresentaram piora em ambos os grupos.

Todavia, houve melhora da percepção da demanda em relação ao estresse, com resultados próximos ao nível de significância $(\mathrm{P}=0,26)$, o que pode estar relacionado ao tamanho amostral relativamente pequeno.

Além disso, nossos resultados não demonstraram efeitos positivos em relação ao bem estar e percepções de controle e apoio social no trabalho. Da mesma forma, um estudo realizado por Bohr (2002) também apresentou resultados semelhantes, visto que não demonstrou efeitos positivos em relação ao estresse (BOHR et al., 2002).

Sabe-se que a intervenção ergonômica baseada na adequação dos postos de trabalho reduz a exposição postural (BARROS et al., 2016). Assim, a redução da exposição postural pode ter levado a uma redução da percepção demanda de trabalho, refletindo nos níveis de estresse no trabalho.

Uma vez que a exposição a fatores de risco e longas jornadas de trabalho expõem os trabalhadores ao maior desgaste mental e físico, esperava-se que a modificação do posto de trabalho reduziria a prevalência de sintomas (MAIZURA et al., 2010).

Uma revisão sistemática recente, sobre a efetividade do ajuste da cadeira para a redução de sintomas musculoesqueléticos, aponta redução da gravidade, intensidade e frequência de dor musculoesquelética (VAN NIEKERK et al., 2012). No entanto deve-se levar em conta que poucos estudos atenderam aos critérios de inclusão desta revisão $(n=5)$ e os estudos não eram específicos para o trabalho de escritório. Assim, os achados obtidos no presente 
estudo apontam evidências de que intervenções voltadas ao posto de trabalho são eficazes para reduzir a percepção de desconforto e a intensidade dos sintomas musculoesqueléticos em trabalhadores de escritório.

Em relação aos resultados positivos obtidos nos níveis de fadiga não foram encontradas na literatura resultados similares a este. Apesar da ausência de estudos relacionando a escala de fadiga à intervenção no mobiliário, notou-se que o ENEDE é sensível a modificações de medidas ergonômicas nos postos de trabalho. Esta escala também já demonstrou ser útil para avaliar os efeitos organizacionais, como a implementação de rodízio de tarefas (KUIJER et al, 2005) e aumento de horas trabalhadas (DE CROON et al, 2006). Assim, a melhora dos sintomas e da percepção de fadiga podem estar relacionadas a menor exposição aos fatores de riscos devido a intervenção ergonômica realizada nos postos de trabalho, uma vez que o ajuste diminui a sobrecarga física e consequentemente a percepção de dor e fadiga.

A ausência de resultados positivos em relação ao estresse e bem-estar podem estar relacionados à restrição da intervenção somente ao posto de trabalho, sem relacionar medidas diretas sobre fatores psicossociais e mentais. Assim, seria relevante incluir medidas de intervenção voltadas também aos aspectos organizacionais do trabalho.

Finalmente, cabe ressaltar que o tamanho amostral foi insuficiente para gerar dados estatisticamente significativos. Neste sentido, cabe apontar que para o tamanho de efeito obtido o tamanho amostral deveria ser de, no mínimo 102 sujeitos. Além disso, avaliações de longo prazo também podem ser relevantes.

\section{CONCLUSÃO}

A intervenção realizada nos postos de trabalho foi eficaz para reduzir a percepção da dor no pescoço, ombros e região superior das costas, e também foi capaz de reduzir os níveis de fadiga captado pela Escala de Necessidade de Descanso (ENEDE). Entretanto para os níveis de estresse e bem-estar no trabalho, não foram alterados pela intervenção realizada no mobiliário. Embora os efeitos benéficos da intervenção tenham sido observados, ainda são necessários estudos sobre esta temática envolvendo amostras maiores de trabalhadores.

\section{REFERÊNCIAS BIBLIOGRÁFICAS}

AHONEM M, Martti, Kuorinka T. Ergonomic Workplace Analysis. Finnish Institute of Occupational Health, 1989

AMICK, Effect of office ergonomics intervention on reducing musculoskeletal symptoms. Spine (2003) 24:2706-2711.

ANDERSON K, Karlehagen S, Jonsson B. The importance of variations in questionnaire administration. Applied Ergonomics (1987) 18(3): 229-232.

ALONSO J, Anto JM, Moreno C. Spanish Version of the Nottingham Health Profile Translation and Preliminary Validity. American Journal of Public Health (1990) 80: 704708.

ALVES MGM, Chor D, Faerstein E, Lopes CS, Werneck GL. Versão Resumido da "job stress scale": adaptação para o português. Revista Saúde Pública (2004) 38 (2): 164-71. 
BARROS ENC, Alexandre NMC. Cross-cultural adaptation of the Nordic musculoskeletal questionnaire. International Nursing Review (2003) 50: 101-108.

BARROS FC. Efetividade de uma Intervenção Ergonômica na Postura, Dor e Desconforto de Trabalhadores de Escritório - Ensaio Randomizado por Cluster e Controlado. Dissertação de Mestrado - UFSCar. Universidade Federal de São Carlos, São Paulo, 2016.

BART NG. A literature review of neck pain associated with computer use: public health implications. The Journal of the Canadian Chiropractic Association (2008) 52(3).

BENEDETTI TRB, Antunes PC, Rodriguez-Añez, Mazo GZ, Petroski EL. Reprodutibilidade e validade do Questionário Internacional de Atividade Física (IPAQ) em homens idosos. Revista Brasileira de Medicina do Esporte (2007) 13 (1):11-16.

BERNARD BP. Musculoskeletal disorders and workplace factors - a critical review of epidemiologic evidence for work-related musculoskeletal disorders of the neck, upper extremity, and low back. Cincinnati: National Institute for Occupational Safety and Health (NIOSH), Centers for Disease Control, Department of Health and Human Services, 1997.

BJORKSTÉN MG, Boquist B, Talbãck M, Edling $C$. The validity of reported musculoskeletal problems. A study of questionnaire answers in relation to diagnosed disorders and perception of pain. Applied Ergonomics (1999) 30(4):325-30.

BOHR PC. Office ergonomics education: A comparison of traditional and participatory methods. Work (2002)19(2):185-191.

BRINK Y, LOUW QA. A systematic review of the relationship between sitting and upper quadrant musculoskeletal pain in children and adolescents. Manual Therapy (2013) 18: 281 $-288$.

CALLAGHAN JP. Do NIRS measures relate to subjective low back discomfort during sedentary tasks? International Journal of Industrial Ergonomics (2010) 40: 165-170.

CRAWFORD JO. The Nordic Musculoskeletal Questionnaire. Occupational Medicine (2007) 57(4):300-301.

COURY HJCG. Trabalhando sentado: manual para posturas confortáveis. São Carlos: Editora UFSCar, 1994.

COSTA BR, Vieira ER. Risk factors for work-related musculoskeletal disorders: a systematic review of recent longitudinal studies. American Journal of Industrial Medicine (2010) 53(3):285-323.

De CROON EM, Sluiter JK, Frings-Dresen MHW. Need for recovery after work predicts sickness absence: a 2-year prospective cohort study in truck drivers. Journal of Psychosomatic Research (2003) 55(4):331-39.

DEYO RA, Battie M, Beurskens AJ, Bombardier C, Croft P, Koes B, Malmivaara A, Roland M, Von Korff M, Waddell G. Outcome measures for low back pain research. A proposal for standardized use. Spine (1998) 15;23(18):2003-13.

DONOVAN DM, Kivlahan DR, Doyle SR, Longabaugh R, Greenfield SF. Concurrent validity of the Alcohol Use Disorders Identification Test (AUDIT) and AUDIT zones in defining levels 
of severity among out-patients with alcohol dependence in the COMBINE study. British Journal of Addiction (2006) 101:1696-1704.

GRANDJEAN, Etienne, Manual da ergonomia adaptando o trabalho ao homem. Porto Alegre: Artes Médicas Sul, 1998.

GONÇALVES CF. Ergonomia e qualidade do serviço bancário: uma metodologia de avaliação. Programa de Pós-Graduação em Engenharia de Produção UFSC, Tese de Doutorado, 1995.

GOULART PM, Blanch JMR, Sahagún MA, Bobsin TS. Questionário de Bem-Estar no Trabalho: estrutura e propriedades psicométricas. Estudos de Psicologia (2012) 29:657665.

HEATHERTON TF, Kozlowski LT, Frecker RC, Fagerstrom KO. The Fagerstrom Test for Nicotine Dependence: a revision of the Fagerstrom Tolerance Questionnaire. British Journal of Addiction (1991) 86(9):1119-1127.

KALINIENE G, Ustinaviciene R, Skemiene L, Januskevicius V. Associations between neck musculoskeletal complaints and work related factors among public servisse computer workers in kaunas. International Journal of Occupational (2013) 26(5):670-81.

KAUSTO J, Miranda H, Pehkonen I, Heliovaara M, Viikari-Juntura E, Solovieva S. The distribution and co-occurrence of physical and psychosocial risk factors for musculoskeletal disorders in a general working population. International Archives Occupational and Environmental Health (2011) 84:773-88.

KISS P, De Meester M, Brackman L. Differences between younger and older workers in the need for recovery after work. International Archives Occupational and Environmental Health (2008) 81(3):311-20.

KUIJER PP, van der Beek AJ, van Dieen JH, Visser B, Frings-Dresen MH. Effect of job rotation on need for recovery, musculoskeletal complaints, and sick leave due to musculoskeletal complaints: a prospective study among refuse collectors. American Journal of Industrial Medicine (2005) 47(5):394-402.

MACDONALD LA, Karasek RA, Punnett L, Scharf T. Covariation between workplace physical and psychosocial stressors: evidence and implications for occupational health research and prevention. Ergonomics (2001) 44:696-718.

MAIZURA H. Job straim amomg Malaysian office workers of a multinational company. Occupational Medicine (2010) 60 (3) 219-224.

MANUAL DE APLICAÇÃO DA NORMA REGULAMENTADORA No 17(NR-17). - 2 ed. Brasília: MTE, SIT, 2002

MINISTÉRIO DA SAÚDE DO BRASIL. Doenças relacionadas ao trabalho - Manual de Procedimentos para os Serviços de Saúde, Cap 19, Editora MS

MINISTÉRIO DO TRABALHO E EMPREGO. Ergonomia. Brasília: MTE/SIR, s.d. Disponível em <http://www.mte.gov.br>.

MÖRL F.; BRADL I. Lumbar posture and muscular activity while sitting during office work. Journal of Electromyography and Kinesiology (2013) 23: 362-368. 
PANAGIOTOPOULOU G, Christoulas K, Papanckolaou A, Mandroukas K. Classroom furniture dimensions and anthropometric measures in primary school. Applied Ergonomics (2004) 35: 121-128.

PARCELLS C, Stommel M, Hubbard RP. Mismatch of classroom furniture and student body dimensions: empirical findings and health implications. Journal of Adolescent Health (1999) 24: 265-73.

PARDINI R, Matsudo S, Araújo T, Matsudo V, Andrade E, Braggion G, Andrade D, Oliveira $L$, Junior $A F$, Raso $V$. Validação do questionário internacional de nível de atividade física (IPAQ-versão 6): estudo piloto em adultos jovens brasileiros. Revista Brasileira de Ciência e Movimento (2001) 9(3):45-51.

PUGH JD, Gelder L, Williams AM, Twigg DE, Wilkinson AM, Blazevich AJ. Validity and reliability of an online extended version of the Nordic Musculoskeletal Questionnaire (NMQE2) to measure nurses' fitness. Journal of Clinical Nursing (2015) 24(23-24):3350-63.

REETA O, Luukkaala T, Manka ML, Nygard CH. A New Approach to Measuring WorkRelated Well-Being. International Journal of Occupational Safety and Ergonomics (2011) 17(4):341-359.

RIJN RM, Huisstede BM, Koes Bw, Burdorf A. Associations between work-related factors and specific disorders of the shoulder-a systematic review of the literature. Scandinavia Journal of Work Environment Health (2010) 36(3):189-201.

SONNE M, Villalta DL, Andrews DM. Development and evaluation of an office ergonomic risk checklist: ROSA - Rapid office strain assessment. Applied Ergonomics (2012) 43: 98-108.

SWAEN GMH, van Amelsvoort LGPM, Bültmann U, Kant IJ. Fatigue as a risk factor for being injured in an occupational accident: results from the Maastricht Cohort Study. Occupational Environment Medicine (2003)60(Suppl. 1):i88-i92.

TAKEKAWA KS, Gonçalves JS, Moriguchi CS, Coury HJCG, Sato TO. Can a selfadministered questionnaire identify workers with chronic or recurring low back pain? National Institute of Occupational Safety and Health. Industrial Health (2015)53(4):340345.

VAN NIEKERK SM, Louw QA, Hillier S. The effectiveness of a chair intervention in the workplace to reduce musculoskeletal symptoms: A systematic review. BMC Musculoskeletal Disorders (2012) 13: 145-58.

WESTGAARD RH, WINKEL J. Guidelines for occupational musculoskeletal load as a basis for intervention: a critical review. Applied Ergonomics (1996) 27(2):79-88

YUE P, Liu F, Li L. Neck/shoulder pain and low back pain among school teachers in China, prevalence and risk factors. BMC Public Health (2012)12:789. 